\title{
Board 56: Retaking Object-Oriented Programming Quizzes for Study Habit Insights and Improvements
}

\section{Prof. Aaron Carpenter, Wentworth Institute of Technology}

Professor Carpenter is an Assistant Professor at the Wentworth Institute of Technology. In 2012, he completed his $\mathrm{PhD}$ at the University of Rochester, and now focuses his efforts to further the areas of computer architecture, digital systems, cybersecurity, and computer engineering education.

\section{James R McCusker PhD, Wentworth Institute of Technology}

James R. McCusker is an Associate Professor at Wentworth Institute of Technology in the Department of Electrical and Computer Engineering. Since joining Wentworth in 2010, he has been heavily involved with an array of interdisciplinary design courses that range from introductory to capstone courses. 


\title{
Retaking Object-Oriented Programming Quizzes for Study Habit Insights and Improvements
}

\author{
Aaron Carpenter and James McCusker \\ \{carpentera1, mccuskerj\}@wit.edu \\ Department of Electrical and Computer Engineering \\ Wentworth Institute of Technology
}

It is often a challenge to gain insight into undergraduate study habits. Students can list the resources at their disposal and can explain the benefits of well-understood techniques (e.g. study groups, individual meetings/tutoring, time management); however, the same students will often ignore the warning signs of academic trouble and resort to poor habits (e.g., web searches for assignment answers). Additionally, students often believe that the knowledge from class doesn't need to be retained beyond one assignment, quiz, or exam, regardless of if they fail the assignment or evaluation.

While it is primarily the students' obligation to seek help when needed, the instructor must also provide avenues for success for the students, by providing structures for student improvement, not just answers and keys. The work presented here describes an effort to (a) seek insights into the student mindset regarding improving their own knowledge and (b) guide students towards reliable resources (videos, slides, websites) to help them with their future studies. As a secondary effect, student grades are improved and students expressed gratitude for the ability to safeguard their grade from a potentially simple mistake.

In a junior-level programming course at Wentworth Institute of Technology, students learning $\mathrm{C}++$ and related concepts had to take a total of nine 10-15 minute quizzes over the course of a 14 week lecture schedule. These quizzes were graded and returned, typically within 2-5 days. If a student earned a grade below 80, they could meet with the instructor within a week and provide new answers for the quiz questions, which, if correct, resulted in a higher grade (maximum of 79). Students were then asked to share how they came to the new/correct answers, whether from websites, videos, peers, or other resources. These resources were not factored into the grade but were used to help guide that student and the entire class to useful resources. If a student were using potentially incorrect or weaker sources, the instructor corrected it. If the sources were strong, they could be shared with other students to help the entire class build a database of useful resources.

A similar, but separate retake policy was also implemented in a junior-level Signals \& Systems course, where students were able to retake examinations, in order to improve their grade and better understand the material. This process is discussed and compared to the retake policy in the programming course. 
This work is still in its preliminary phase, with this paper describing the pilot run of the retake process. Despite the early time-frame, this work will share several key insights useful to instructors in programming and in unrelated fields. First, a statistical study in how students took advantage of the opportunity to improve their grades and what sort of impact it had on that student's grades, in both the short and the long-term of the course (for example, did their future quiz grades improve before utilizing the retake policy). At the same time, quiz grades were meant only an assessment tool, and student knowledge retention is explored, as revealed through a final quiz and survey questions. Next, this work discusses what resources the students were using for both individual concepts and across the entire course. This will help instructors understand to where current students turn when they need help or additional information, as well as provide potentially useful tools to instructors of similar courses. Further insights gained through the analysis is also shared, both qualitatively and quantitatively, to help prepare students for better decision making regarding studying and resources.

\section{Introduction}

Students have a wealth of resources at their disposable for gaining knowledge and information pertaining to a class (e.g., lecture notes, books, peers, instructors, the Internet, etc.). Though when faced with an academic challenge, as most instructors will share quantitatively or anecdotally, students primarily turn to the Internet to search for guidance.

Of course, the web should serve as a valuable tool for their education and for their ability to learn new skills and knowledge beyond their time as undergraduates. This is increasingly problematic, though, in the area of computer programming, as students rely primarily on the web for syntax

and exemplar code. The key to programming skills lies not only in the ability to write algorithms, debug, and write proper syntax but also the ability to find resources and properly utilize them in a new code file. For example, if a student is going to use an external $\mathrm{C}++$ library, simply finding a YouTube tutorial video may be insufficient. Students need to identify proper sources and read documentation to internalize the information for future use.

At the same time, students will sometimes hesitate to use their professors or instructors as resources. Instructors can not only share knowledge of the course material but also guidance in study habits and long-term learning.

The authors implemented a retake policy in an effort to (a) help students gain and retain knowledge within a programming course, (b) evaluate how students are studying when they make mistakes on quizzes, and (c) help students' knowledge and grades.

Students in an object-oriented programming course in the Electrical and Computer Engineering department at Wentworth Institute of Technology were given nine quizzes over a 14-week lecture period. Eight of these quizzes were open to be revisited by any student that received a score less than 80 . These students could meet with the instructor individually and explain their mistakes. They would then be asked what they used to study for the retake. Data were gathered regarding their grades, habits, and the overall impact it had on their grade and knowledge retention.

In a comparison, another professor in the same department has used retakes of exams to help 
students demonstrate growth. The time dedication for the instructor for this attempt was significantly less than the quiz retake and had a separate set of guidelines that can be adapted to better suit the needs of the class and instructor.

Both approaches allow insight for the instructor that could then provide meaningful feedback on student study habits and provide useful tools to the entire class. For example, certain websites were publicly described as beneficial, while others were described as potentially detrimental. This helped steer students towards better studying decisions, which in turn, will lead to stronger life-long learning techniques as new skills are required.

The courses were taught at Wentworth Institute of Technology, which is a STEM-focused, undergraduate-centric university in Boston, Massachusetts. The student body primarily studies engineering, applied sciences, and architecture and construction fields. Class sizes are typically small, averaging around 20-25 students per class, with no teaching assistants.

In Section 2, related work in the field will be compared and contrasted to this work. Sections 3 and 4 will describe the experimental procedures, data, and analyses. Section 5 will provide lessons learned, recommendations, and conclusions drawn from the investigation.

\section{Related Work}

Students often lack the understanding of their own study habits [6], and thus the quiz retake policy is aimed to reinforce their good habits and dissuade them of bad ones.

The use of quizzes for student assessment and reinforced learning is certainly a well-known technique inside of college classrooms. Various studies have shown how to use online learning management systems to create digital quizzes [2,3]. Others study how often to give quizzes and their use as a replacement to other class work $[8,5]$, including evaluations comparing exam grades.

There is a body of work that uses quiz retakes as a way to reinforce concepts and improve long-term retention $[1,4,2,3,9,7]$. Each of these had related but not directly comparable approaches, goals, and outcomes to the study presented here. The most similar work asks similar questions within a non-programming-related course and found that few students used the internet [9]. Another similar work explored the use of the exam retakes outside of class and without instructor feedback [4]. That work forced students to attempt to retake assessment or risk losing points and found that it improved grades on future exams and helped to keep students engaged in the course. The investigators of this previous work also did not require face-to-face interaction between the students and instructors but allowed the students to work outside the class and submit new answers. In the work presented here, all students must answer questions live to the instructor.

Other researchers explored the use of multiple retakes, rather than a single retake attempt [2, 3, 1]. These multiple attempts were often timed and tracked. In the work presented here, only a single attempt is allowed, and no time was tracked. Instead, the focus was on fixing possible gaps in study habits. 


\section{Quiz Retake Procedure and Data}

\subsection{Object-Oriented Programming Course: Quiz Retakes}

This work includes data from a single semester (two sections) of an Object-Oriented Programming course at Wentworth Institute of Technology. The two sections held approximately 30 students each, for a total of 60 students (the actual number varied based on withdrawals near the end of the semester). At the time of publication, this policy is being used again in the same course, although this data is withheld as the semester is incomplete. The course curriculum includes standard topics, such as conditionals, loops, pointers, classes, inheritance, polymorphism, basic data structures, and algorithmic design. After a topic was covered in lecture, students had to take an in-class quiz based on the lecture and in-class activities. In the fall of 2018, the class included eight 10-15 minute quizzes, with a ninth quiz serving as a review/cumulative quiz to re-examine the questions students struggled with on the previous quizzes. The eight quizzes were then graded and handed back (typically within 2-5 days). Students were allowed to record their grade and any markings from the quiz (most chose to take pictures), and the quizzes were then collected. These quizzes did not include writing code, but focused on concepts, reading and understanding code, and algorithms.

The average of the quiz scores varied, ranging from $56 \%$ to $90 \%$, depending on the material. Figure 1a details the averages. The worst averages were the quizzes on class syntax and structure, pointer analysis and manipulation, and inheritance of private variables. However, each quiz had at least six (out of 60) students eligible to retake the quiz (their scores were under 80). Figure 1b shows how many students were eligible to improve their score and how many actually did so.

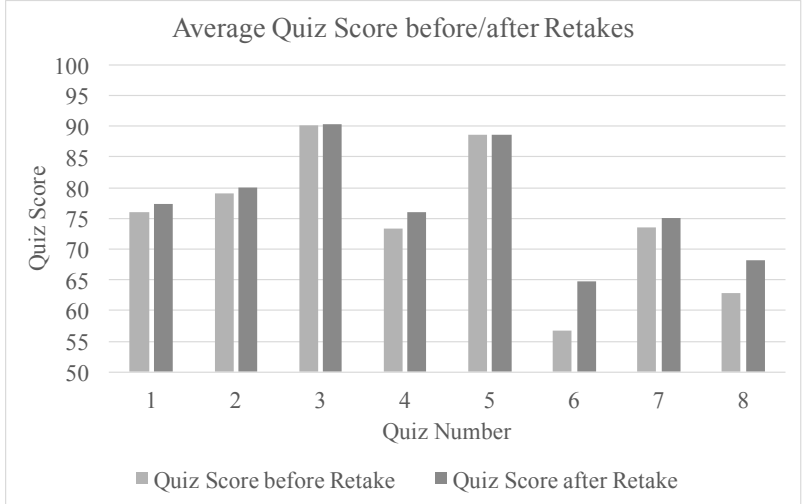

(a) Average Quiz Scores

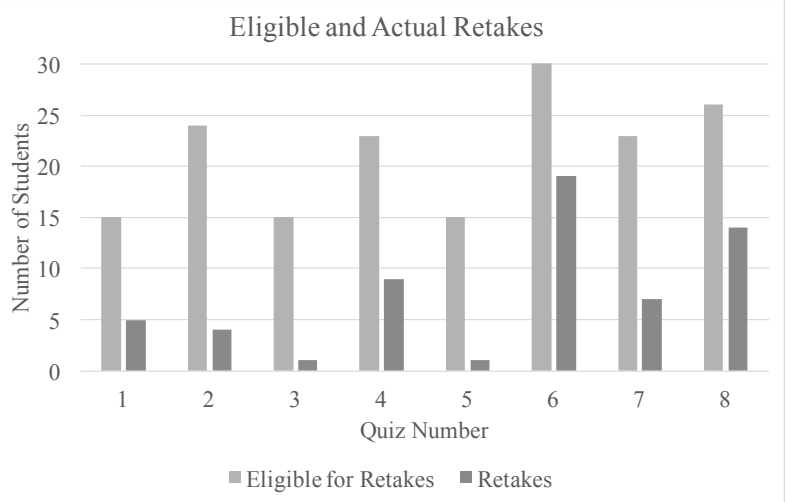

(b) No. of Eligible and Actual Retakes

Figure 1: Statistics for average quiz score and the number of eligible and actual retaken quizzes.

Within a week of the quizzes being handed back, the students could meet with the instructor and privately correct their mistakes. As the quizzes were marked with indications of point-deductions, students did have some guidance of where to look for their corrections. Students then described their new answers as the instructor wrote the students' changes on the quiz. If the new answers were incorrect, the instructor indicated where the students' were still mistaken (but no additional 
points were given for that answer). The quiz was regraded with the new answers, with 79 being the maximum score. This was to be fair to the students who received an 80 or higher and thus were not eligible for retaking the quiz. Students could only retake each quiz one time, regardless of the grade of the second attempt. This proved largely inconsequential, as most students maximized their grade after the first retake. Future exploration will include the possibility of multiple retakes.

The new grade was dependent on the correctness and quality of the new answers as well as their original score. For example, a student that received a 25 on the quiz, but then got all answers correct during the retake would receive a 70 to reflect both their initial low score and their new understanding. Figure 1 illustrates the original and post-retake averages and the number of eligible retakes.

After revisiting the quiz, students were asked of their sources for studying the quiz (after the initial grading cycle). These sources were gathered qualitatively, and then coded and converted to quantitative data. The sources are listed in Figure 2. The sources themselves were not factored into the grading of the retake. However, if the student cited a source that was deemed inappropriate or less trustworthy, the instructor would discuss other possible sources. Additionally, good sources were shared with other students or the class as a whole to make the study resources more widely known. Over time, this helped eliminate weaker sources and help students identify good resources for future studying.

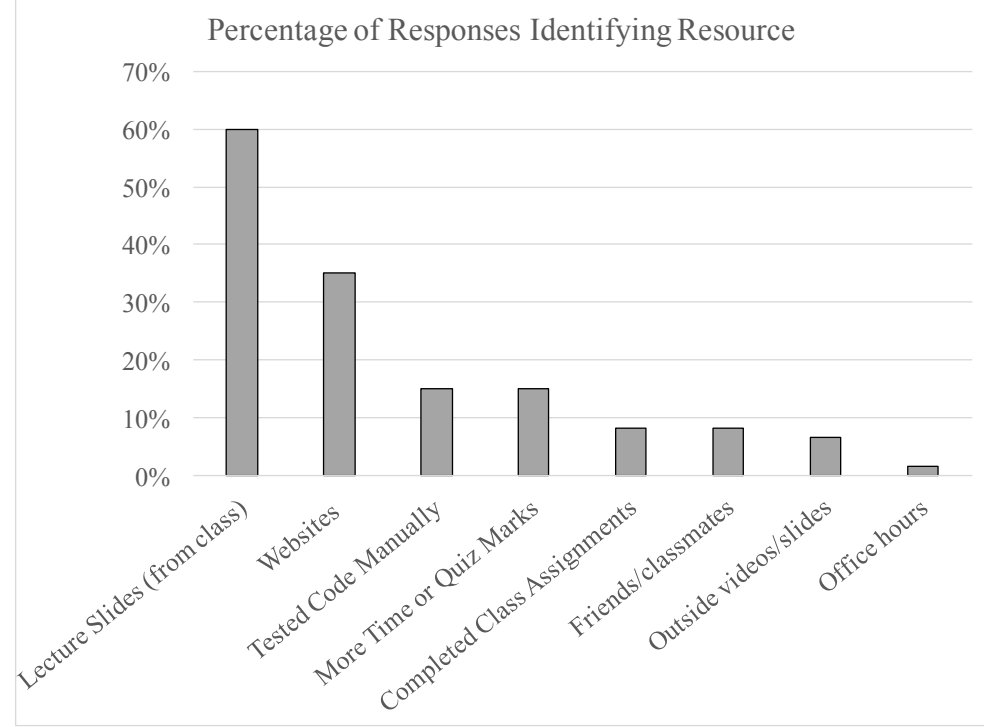

Figure 2: The reported sources of studying for the retake. Students could give more than one resource, so the numbers do not sum to $100 \%$.

Quiz retakes were only allowed within a week of the students seeing their grade, and only a single retake was allowed per quiz. All quizzes were eligible for the retake policy except the final one. The last quiz was used instead as a cumulative quiz final, counting the same as the other eight, but reviewing material from all 14 weeks of lectures. This quiz also allowed insight into what students were retaining through the course. In particular, the three questions chosen were exact copies of questions from the lowest-scoring quizzes. Student scores were then compared to the 
scores from the original quizzes using the ability to or choice to retake as the lens with which to organize. Figure 3 shows the results of this comparison. True correlation was not significant here due to the small sample size.

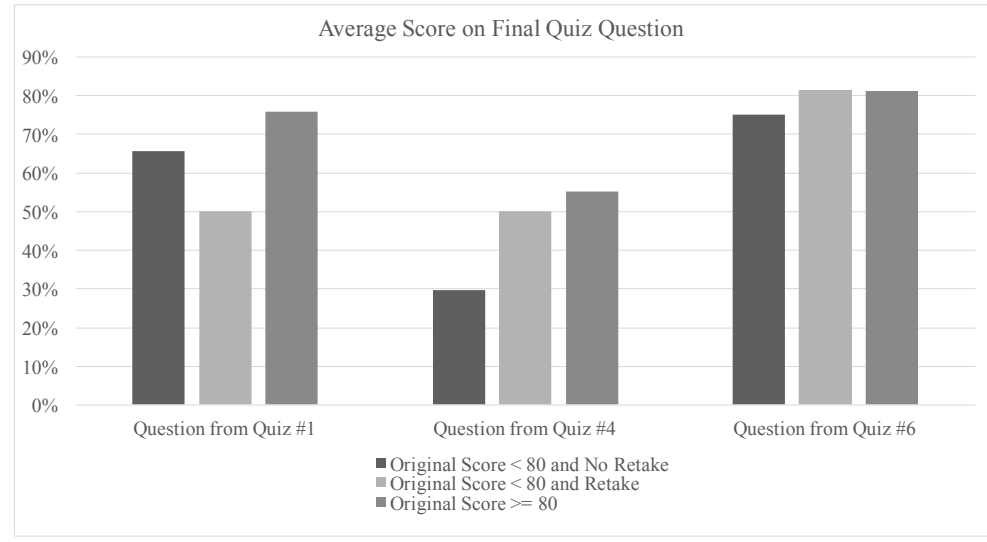

Figure 3: The final quiz revisited questions from past quizzes. The graph examines whether the retake of the original quiz had an impact on whether the students did better or worse on that question.

\subsection{Signals and Systems Course: Exam Retakes}

Although effective in enhancing student understanding of material and improving student grades, approach presented in Section 3.1 requires the course instructor to incur a significant investment of time. An alternate approach was utilized in the course Signals and Systems that aimed to improve student understanding but limit the investment in time incurred by the course instructor (a different instructor than the one teaching the previously discussed programming course). This particular course enrolled approximately 30 students and addressed topics such as signal operations, system and signal classification, time domain analysis, Laplace transformations, Fourier series and transformations, and filtering. In the summer of 2018, the course involved numerous 15 minute quizzes and three 120 minute exams throughout the semester. Grades typically ranged from $60 \%$ to $100 \%$ on quizzes and between $50 \%$ and $95 \%$ on exams.

In any instance (quiz or exam) where the class average fell below 75\%, a retake was offered. To ensure fairness, the retake was offered to the entire class and the final score was the average of the retake and their original attempt. The structure of the retake was in the form of a take home exam wherein students would be allotted 24-48 hours to work through the exam independently. In order to encourage students to reevaluate their prior attempts, provide insight for the course instructor pertaining to students' understanding of topics, and to limit disallowed collaboration, students were required to provide a detailed description of why the prior attempt was incorrect, what incorrect assumptions were made, and the logic behind the answer submitted in the retake. In addition, to both encourage students to check their work and to limit the time required by the course instructor to regrade the exams, students were informed that there would be no partial credit provided on any questions. Based on both student feedback and student performance on a 
cumulative final exam, this exercise proved to be a valuable tool to enhance student understanding.

Both of the discussed retake policies will be further explored and examined in future iterations of the courses. As more data are gathered and further insights gained, they will be disseminated accordingly.

\section{Quantitative and Qualitative Analyses}

Impact on Knowledge Retention: Figure 3 illustrates the average score on each question of the final quiz. The final quiz included three questions, each from a particularly low-scoring quiz portion. Question 1 (from quiz \#1) was regarding operators and data types; question 2 (from quiz \#4) included pointer manipulation and referencing/dereferencing; and question 3 (from quiz \#6) assessed class and function syntax. Each of these questions was among the most penalized for all eight quizzes.

For each of the latter questions, the students that scored poorly in the original quiz did better than their classmates that chose not to retake it. Meanwhile, the cohort that was already getting above $80 \mathrm{did}$ predictably well on all three questions. This implies that the retake policy and procedure may have helped those students to fill in gaps in their knowledge and assist in retaining the information. For question \#1, there were only five students that decided to retake it, and two of those got 0 on that question, which brought the average down significantly. Any future work will need to take into consideration the number of retakes to get a more reliable conclusion.

Overall, students that took advantage of multiple retakes did improve their overall understanding of the concepts, as can be only anecdotally reported from conversations and non-quiz assignments as quiz concepts were only used once (other than the final quiz).

Impact on Study Resources: In the first weeks of the semester, as students relayed their resources to the instructor, a number of code forum and tutoring websites were cited. The instructor pointed out that websites linked to companies or academic institutions were a better source for reliable information. Sites like stackoverflow, chegg, coursehero, and random sites from around the web were cautioned against (with reasons provided to students), while other sources like academic videos, www.cplusplus.com, and even www.geeksforgeeks.org were cited as more positive resources.

In the end, students relied on two major sources for their studying: class PowerPoint slides and these websites. Other students wrote code and tested it using debuggers (gdb was encouraged), which was encouraged as a legitimate way to learn. Others simply said they needed a second look at the quiz or the grading marks gave indicators of a mistake that could be quickly fixed.

The interaction with the instructor was useful to help steer students away from conventionally unreliable sources. After 1-2 retakes, most students had abandoned the less reliable sources in favor of class notes, in-person discussions with classmates and the instructor, and instructor-approved websites. This prevented students from mistakenly repeating old habits. 
Impact on Final Grades: One might also wonder how this policy affected the individual grades for the students in the course. Quizzes were worth $15 \%$ of the final grade, with eight of these quizzes being included in the retake policy; thus, as each quiz is worth $1.67 \%$, the total portion was about $13 \%$. On average, students raised their final grade by about .66 points (out of $100)$, with two students raising their grades by over 2 points.

Qualitative Comments: In addition to the quiz activities, students were asked to fill out a brief open-ended survey regarding the quiz retake policy. Questions included: "Did you take advantage of the policy" with follow-ups of why or why not? Students that responded "yes" were also asked to describe, despite their initial reasons, what were the main outcomes of doing the retakes. The qualitative comments were coded for keywords and then quantized. Figure 4 provide the reasons students chose to either retake or not retake the quizzes, as categorized by the investigators from open-ended responses. Figure 5 examines the outcomes students cited from the retake policy.

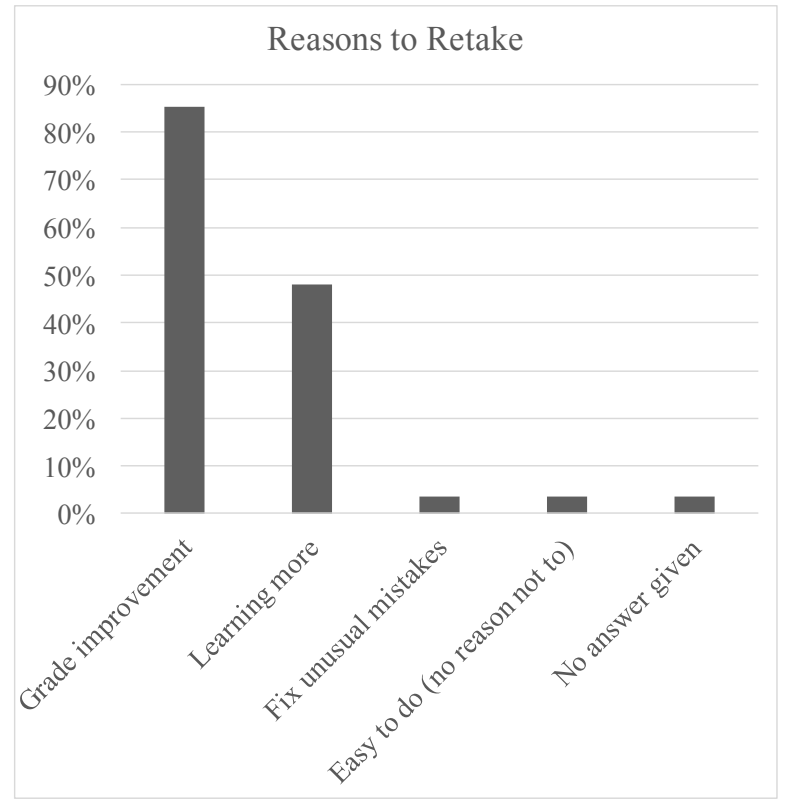

(a) Reasons to Retake

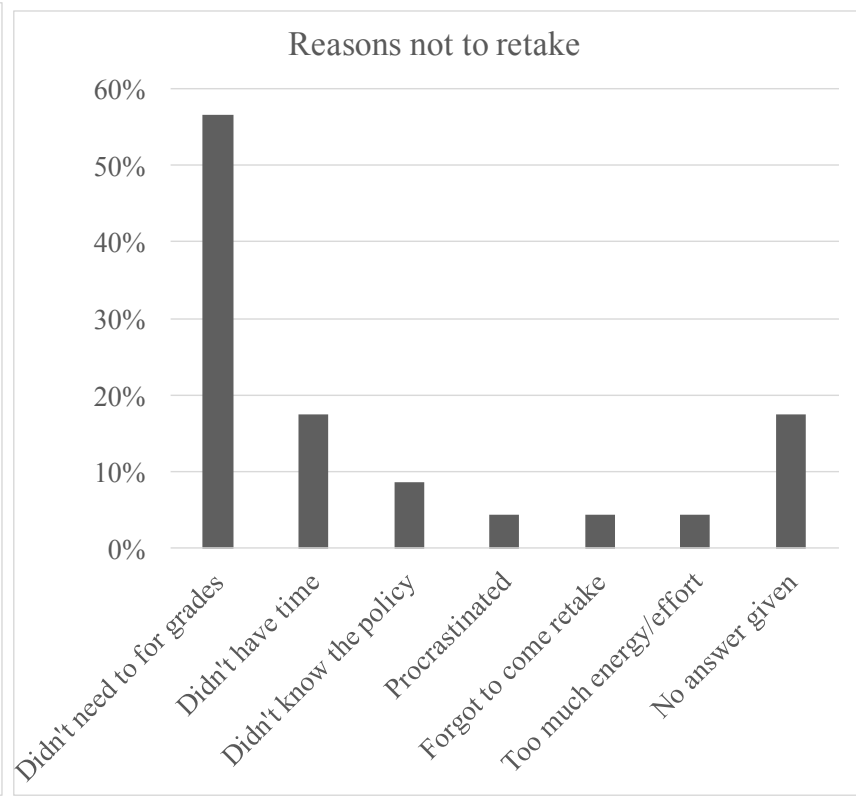

(b) Reasons to Not Retake

Figure 4: (a) The reasons students gave for retaking quizzes. (b) The reasons students gave for not retaking quizzes. Students could give more than one reason, so numbers do not add to $100 \%$.

In general, students chose to use the retake policy for the major reasons one would expect: better grades and helping to learn. Surprisingly, the more cited outcome for students was learning, more so than grades. In other words, students came in to get better grades, but more notably left more satisfied and impacted by their increased knowledge and skill. Figure 5 shows more detail on these outcomes. The results in the figure demonstrate that students may think that better grades are the goal, but actually the more important takeaway is a self-described better understanding and improved learning of concepts and skills. Similarly, the reasons to not retake quizzes were somewhat expected, including not having time, not needing to because of already high grades, and not knowing of the policy. 


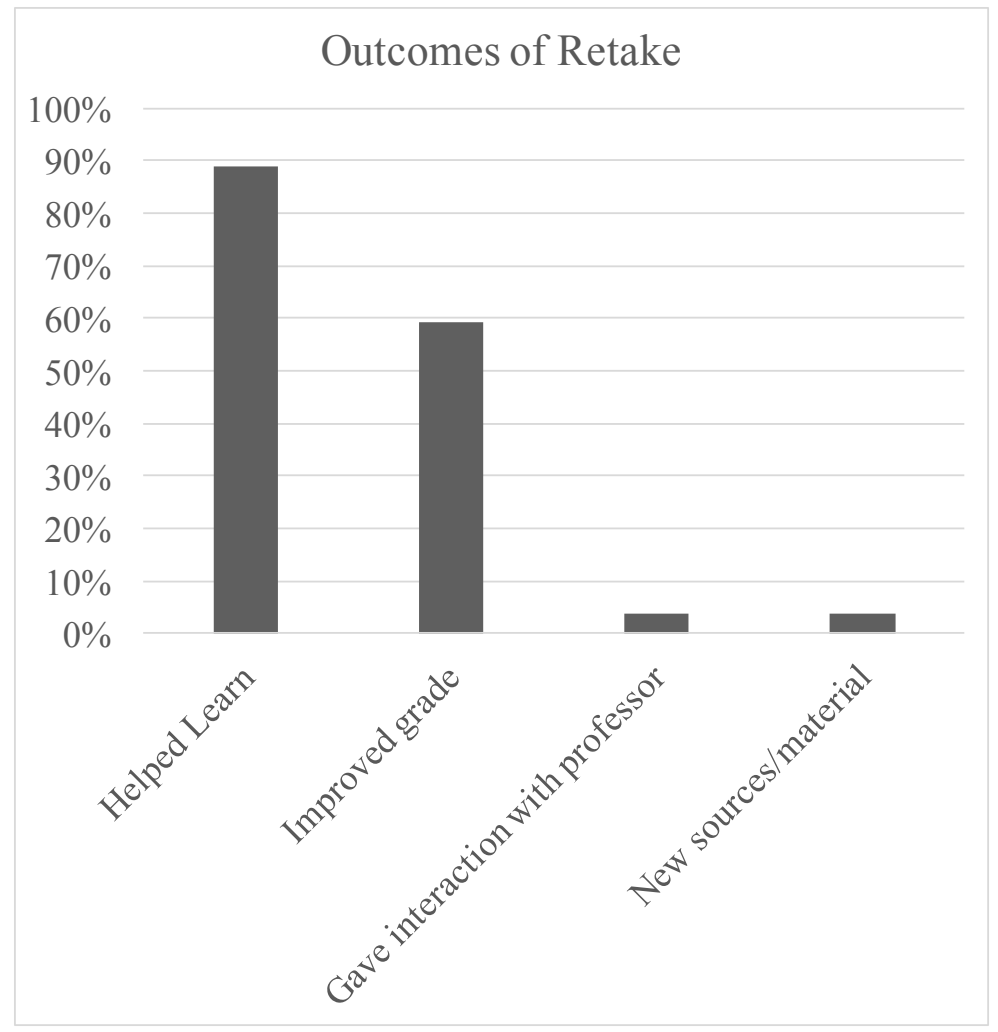

Figure 5: The reported outcomes of utilizing the retake policy. Students could give more than one reason, so numbers do not add to $100 \%$.

The second method described here has no quantitative data, but anecdotally, the students in the course were happy to benefit from a second attempt and the instructor felt more confident in their abilities and grades after the retake. It also took less time than the described quiz retake process based on the omission of partial credit as a component of the grading scheme.

\section{Lessons Learned and Conclusions}

\subsection{Lessons Learned}

The quiz retake policy provides both the student and instructor with information useful to the class' development. For students, it provides higher grades, personal attention from the instructor, guided study suggestions, and the ability to learn from mistakes. Instructors are provided a new avenue to reach out to struggling students and remove communication barriers and also gain insights into how students are studying. This allows the instructor to correct possibly dangerous habits of random web searches and steers students towards a more appropriate life-long learning skill-set.

At the conclusions of the pilot semester of this quiz retake policy, there are several useful insights for instructors: 
- Be sure to clearly and widely advertise and encourage the retake procedure. Many students ignored the initial announcements, resulting in fewer students taking advantage of it in the early semester. Early indications for the new semester show students take advantage more consistently given better advertising.

- Students will sometimes try to simply memorize the answer or bring in study notes to read from, particularly for definitions or explanations. It is suggested to prohibit this in the policy language and to ask for the answer to be done in the student's own words extemporaneously.

- Having a one-week deadline for each retake is a simple way to keep students active throughout the semester. Not doing so could be dangerous for both the student that procrastinates and for the instructor who needs to manage their own time.

- After the one-week retake period has elapsed, review the quiz during a lecture to make sure those that did not qualify for the retake have the opportunity to learn from their mistakes as well. Otherwise, the people who received a grade under 80 get information that the remaining students do not.

- Note that while this will help students in general, it will not be a substitute for larger-scale tutoring for students who may require more attention. It is not meant to be a substitute but rather a supplement to help scaffold independent student learning and programming.

- Marking the quizzes does give a hint to students about their mistakes. This could be positive or negative, depending on the instructor's approach.

- The time allotment for these quiz retakes can be overwhelming if the scores for many students are low. It may be worth it to specify times or make appointments so that office hours do not get used primarily for retakes. This will require more instructor time and effort, but it will free office hours for other interactions. The second discussed policy, regarding exams, showed similar benefits with less instructor time.

\subsection{Conclusions}

Through a 14-week lecture schedule at Wentworth Institute of Technology, approximately 60 students in an Electrical and Computer Engineering department were subject to the use of a quiz-retake policy in the course Object-Oriented Programming. By allowing students the opportunity to retake low-scoring quizzes, students benefit from increased instructor interaction, higher grades, and better structured study habits. At the same time, instructors benefit from the increases interaction with students and insight into their default study resources, thus allowing the instructors to eliminate poor resources and promote more positive ones. Student feedback was unanimously positive, even for those that did not take advantage of the opportunity. Most importantly, students saw the value in the retake policy as a means to improve their knowledge, not just their grade. The instructor gained valuable information to help better teach the entire class, not just individual students. While it does take more time and effort for students and instructors alike, similar policies in future classes can help to better scaffold the learning for a 
student body, improving knowledge retention and satisfaction, better preparing the students for a career in the course's field.

\section{References}

[1] J. E. Bluman, K. Purchase, and C. T. Duling. Daily review quizzes: A hindrance or a help? In 2011 ASEE Annual Conference \& Exposition, June 2011.

[2] K. Davis. Improving motivation and knowledge retention with repeatable low stakes quizzing. In 2009 Annual Conference \& Exposition, June 2009.

[3] K. A. Davis. Using no-stakes quizzing for student self-evaluation of readiness for exams. In 2011 ASEE Annual Conference \& Exposition, June 2011.

[4] W. Guo and V. Shekoyan. Facilitation of student-centered formative assessment using reflective quiz self-corrections in a calculus physics course. In 2014 ASEE Annual Conference \& Exposition, June 2014.

[5] J. H. Allen III, J. Fulcher, and S. I. Selvaraj. Assessment of student learning in undergraduate engineering courses using quizzes in lieu of homework. In 2017 ASEE Annual Conference \& Exposition, June 2017.

[6] B. Kucharski, A. Carpenter, J. Giblin, and M. Ergezer. Work in progress - the undergraduate perspective: How to survive an undergraduate engineering program. In 2018 ASEE Annual Conference \& Exposition, June 2018.

[7] B. Paff. Effect of test retakes on long-term retention. Master's thesis, University of Wisconsion, 2012.

[8] B. Stuart. Professor and student response to the daily quiz. In 2005 Annual Conference, June 2005.

[9] S. B. Velegol and K. S. Jackson. Quiz re-takes: Which students take advantage and how does it affect their performance? In 2015 ASEE Annual Conference \& Exposition, June 2015. 